\title{
SYNTHESIS AND BIOLOGICAL EVALUATION OF NOVEL PYRAZOLO[3,4-D]PYRIMIDINE DERIVATIVES OF EXPECTED ANTICANCER ACTIVITY
}

\author{
Waheed Ali Zaki ${ }^{1}$, Mohamed Alswah ${ }^{*}$, Mohamed M. Elsebaei ${ }^{1}$, Ashraf Hassan \\ Bayoumi $^{1}$ \\ ${ }^{1}$ Pharmaceutical Organic Chemistry Department, Faculty of Pharmacy (Boys), Al- \\ Azhar University, Cairo 11884, Egypt
}

*Corresponding author: drmohammedalswah@azhar.edu.eg

\begin{abstract}
A series of novel pyrazolo[3,4- $d]$ pyrimidine derivatives have designed and synthesized in synthetically useful yields. All the new synthesized compounds were biologically evaluated in vitro for their cytotoxic activities against a panel of three cancer cell lines namely, HepG-2, MCF-7, and HCT-116. The results of cytotoxic evaluation indicated that compound (9) exhibited the most prominent cytotoxic effect against all tested cell lines with $\mathrm{IC}_{50}$ values ranging from (4.03- 6.18) $\mu \mathrm{M}$ comparable to that of doxorubicin as a control drug $\left(\mathrm{IC}_{50}\right.$ values of 8.17 and $\left.9.27 \mu \mathrm{M}\right)$. In particular, compounds $(\mathbf{8 , 9}, \mathbf{1 1})$ and (12) exhibited higher intercalative activity with $\mathrm{IC}_{50}$ value of $30 \mu \mathrm{M}$ than doxorubicin $(31 \mu \mathrm{M})$.
\end{abstract}

Keywords: Anticancer, pyrazolopyramidine derivatives, Topoisomerase II, DNAintercalator. 


\section{I-INTRODUCTION:}

Cancer remains one of the most common causes of death throughout the world and thus the development of potent and more effective anticancer agents represents one of the most important challenges in therapeutics due to the unrivaled pathophysiology of tumors and the predictable emergence of resistance to medication (Thun et al. 2010). Classical methods for cancer treatment including radiotherapy, chemotherapy, and immunotherapy with their own limitations.

Anticancer drugs have been classified into two main target types: the first one is drugs that target DNA, RNA, or proteins. The second target includes other elements involved in the carcinogenesis process, such as the immune system, the endothelium and the extracellular matrix. Most classical chemotherapeutic agents interact with tumor DNA (Espinosa et al. 2003). Compounds that affect DNA include groove binders, alkylating agents, DNA intercalators, and topoisomerase inhibitors (Hurley 2002).

Topoisomerases are important nuclear enzymes, which play a pivotal role in DNA replication, transcription, chromosome segregation, and recombination. There are two fundamental types of topoisomerases; (a) topoisomerase I (Topo I), which is responsible for cleavage, relaxing, and releasing of one strand of the DNA duplex, (b) topoisomerase II (Topo II), which cleaves both strands of the DNA helix simultaneously to remove DNA super coiling (Wang 2002). These enzymes covalently bind to DNA helix via tyrosine residues in the active site. These linkages are transient and easily reversible, and the covalently bound structure is known as the cleavable complex (Denny 2004). Accordingly, topoisomerases are considered as crucial targets for cancer chemotherapy treatments (Pommier et al. 2010). Topoisomerase inhibitors block the ligation step of the cell-cycle, generating single and double stranded breaks that harm the integrity of the genome (Mlcochova et al. 2018). Introduction of these breaks subsequently leads to apoptosis (Kaina2003).

Some anticancer drugs targeting Topo II inhibit the enzymatic activity as a primary mode of action and are known as catalytic Topo II inhibitors (Nitiss 2009). Another type of Topo II-targeting drugs, including intercalating drugs, interfere with the enzyme's cleavage and rejoining activities by trapping the cleavable complex and thereby increasing the time of the transient Topo II-catalyzed DNA breaks. These drugs are referred to as Topo II poisons because they convert the Topo II enzyme into a DNAdamaging agent (Pommier et al. 2010, Nitiss 2009).

These class of drugs act either by topo poisoning via inter-chelation with DNA as doxorubicin (Liu et al. 1989), a msacrine (Sung et al. 2005) and mitoxantrone (Shenkenberg et al. 1986). On the other hand, drugs act as catalytic inhibitors of Top-II as TSC24 (Huang et al. 2010), HY-1 (zhao et al. 2011) and compound (I) (Islam et al. 2017).

DNA Intercalators and Topo II poisons share three common essential structural features. The first one is a planar polyaromatic system (chromophore) which is sandwiched between DNA base-pairs (Laponogov et al. 2013). The second feature is a cationic species, interacting with the negatively charged phosphate group of DNAs. The 
cationic center may be an amino or nitrogen containing heterocyclic group, which can be protonated at physiological $\mathrm{pH}$ (Lee et al. 2017). The third feature is a flexible side chain that anchors DNA (Bailly et al. 2012) (Figure1).

On the other hand, pyrazolopyramidine moieties have anticancer activities (Schenone et al. 2014). In addition, the discovery of new therapeutic DNA intercalators

for the treatment of cancer are considered one of the most important goals in the field of medicinal chemistry (Varrica et al. 2018). Pyrazolopyramidine analogs exhibited excellent anticancer activities through DNA intercalation (Cheng and Robins, 1956) (El-Enany et al., 2010). Pogorelčnik and co-workers optimized the first antitopoisomerase II pharmacophore belonging to pyrazolo[3,4- $d]$ pyrimidine scaffold performing systematic screening to predict the bioactivity between molecule and drug target. Compound (II) was a result of this high-throughput screening (HTS) and efficacious candidate in the series of pyrazolo[3,4- $d]$ pyrimidine which showing promising anticancer activities in hepatocellular carcinoma (HepG2) and breast cancer (MCF-7) cell lines with mean $\mathrm{IC}_{50}$ value of $1.30 \mu \mathrm{M}$. besides, its topoisomerase inhibition (Pogorelčnik et al. 2015) (Figure2).

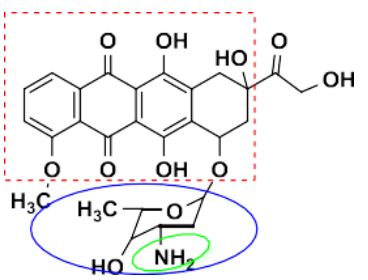

Doxorubicin

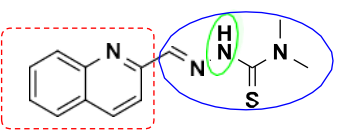

TSC 24

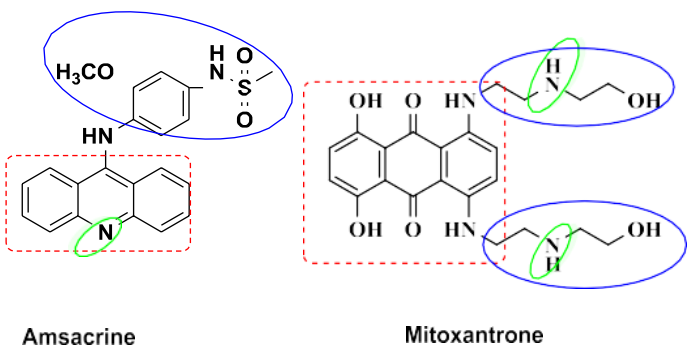

Chromophore
HY-1

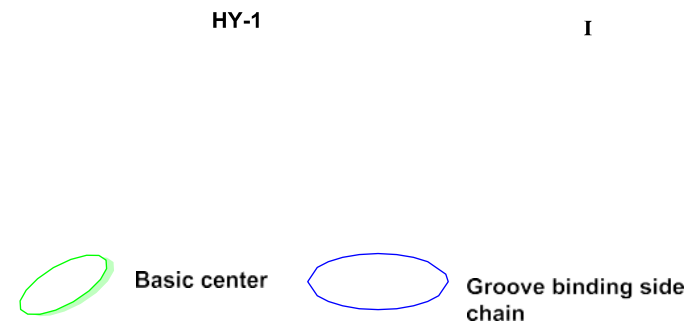

(Figure 1). DNA intercalators and their basic pharmacophoric features 


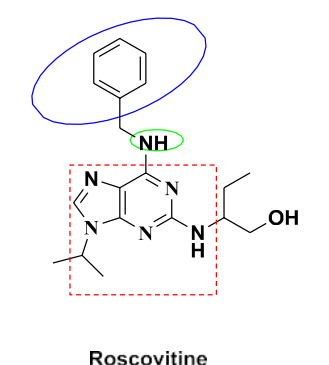

Roscovitine

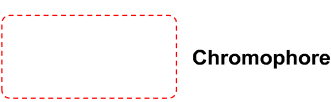

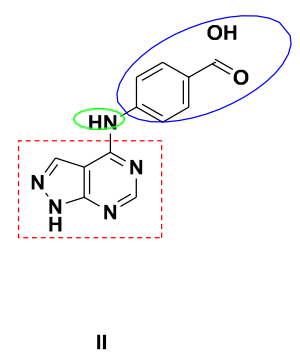

Basic cente

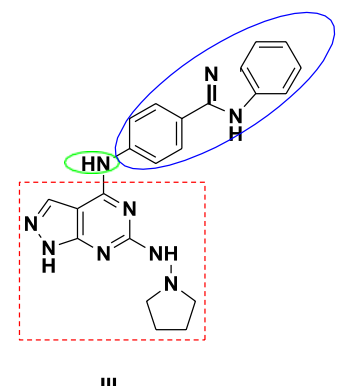

III

Groove binding side chain

(Figure 2). Pyrimidine derivatives as topoisomerase II Inhibitors

Compound (III) proved to be the most active and efficacious candidate in this series, with mean $\mathrm{IC}_{50}$ values of $1.30 . \mu \mathrm{M}$ Further biological evaluation suggested that this compound induce apoptosis and inhibit human topoisomerase (Topo) II (Singla et al. 2017). On the other hand, roscovitine, belongs to the family of purine and is used for the treatment of lung cancer with $\mathrm{IC}_{50}$ value of $2.7 \mu \mathrm{M}$ (Whittaker et al.2004).

Therefore, on the basis of previously above-mentioned findings and in resumption of our previous efforts in the design and synthesis of new anticancer agents (Gaber et al. 2018), we report the design, synthesis and anticancer activities of new pyrazolo[3,4- $d$ ]pyrimidine derivatives. These derivatives were designed based on the main pharmacophoric features of DNA intercalators.

\section{II- Results and Discussion:}

\section{II.1 Chemistry:}

The designed compounds were synthesized as outlined in schemes 1 and 2 . Ethoxymethylene malononitrile (1) (Ding et al. 2012) was allowed to reflux with commercially available phenyl hydrazine in ethanol to produce 5-amino-1-phenyl- $1 \mathrm{H}$ pyrazole-4-carbonitrile (2) (Cheng et al. 1956). Compound (2) underwent a partial hydrolysis using alcoholic sodium hydroxide to produce carboxamide derivative (3) (He et al. 2011).6-methyle-1-phenyl-1,5-dihydro-4H-pyrazolo[3,4-d]pyrimidine core (4) (Miyashita et al. 1990) was formed from the reaction of compound (3) with ethylacetate, sodium ethoxide in absolute alcohol with subsequent chlorination using phosphoryl trichloride to afford compound (5) (Miyashita et al. 1998). The obtained compound (5) was refluxed with hydrazine hydrate to afford 4-hydrazinyl-6-methyl-1-phenyl-1,5dihydro-4H-pyrazolo[3,4- $d$ ]pyrimidine (6) (Gaber et al.2018). Compound (4) using phosphorous pentasulfide in pepridine also we used lawesson reagent in THF afforded unappropriated yield (9)(Elsebaei et al., 2019; Mancy et al., 2019)so that the ${ }^{1} \mathrm{H}-\mathrm{NMR}$ 
spectrum of compound (6) revealed singlet signal at $14.32 \mathrm{ppm}$ corresponding to $\left(\mathrm{NH}_{2}\right)$ proton and compound (6) singlet signal at $13.65 \mathrm{ppm}$ corresponding to (NH)proton. Furthermore, compounds (7) and (8) were prepared by cyclo-condensation of compounds (6) with 2,6-dichlorobenzaldahyde and cinnamaldehyde in absolute ethanol with catalytic amount of glacial acetic acid to give the corresponding hydrazones (7) and (8) respectively.

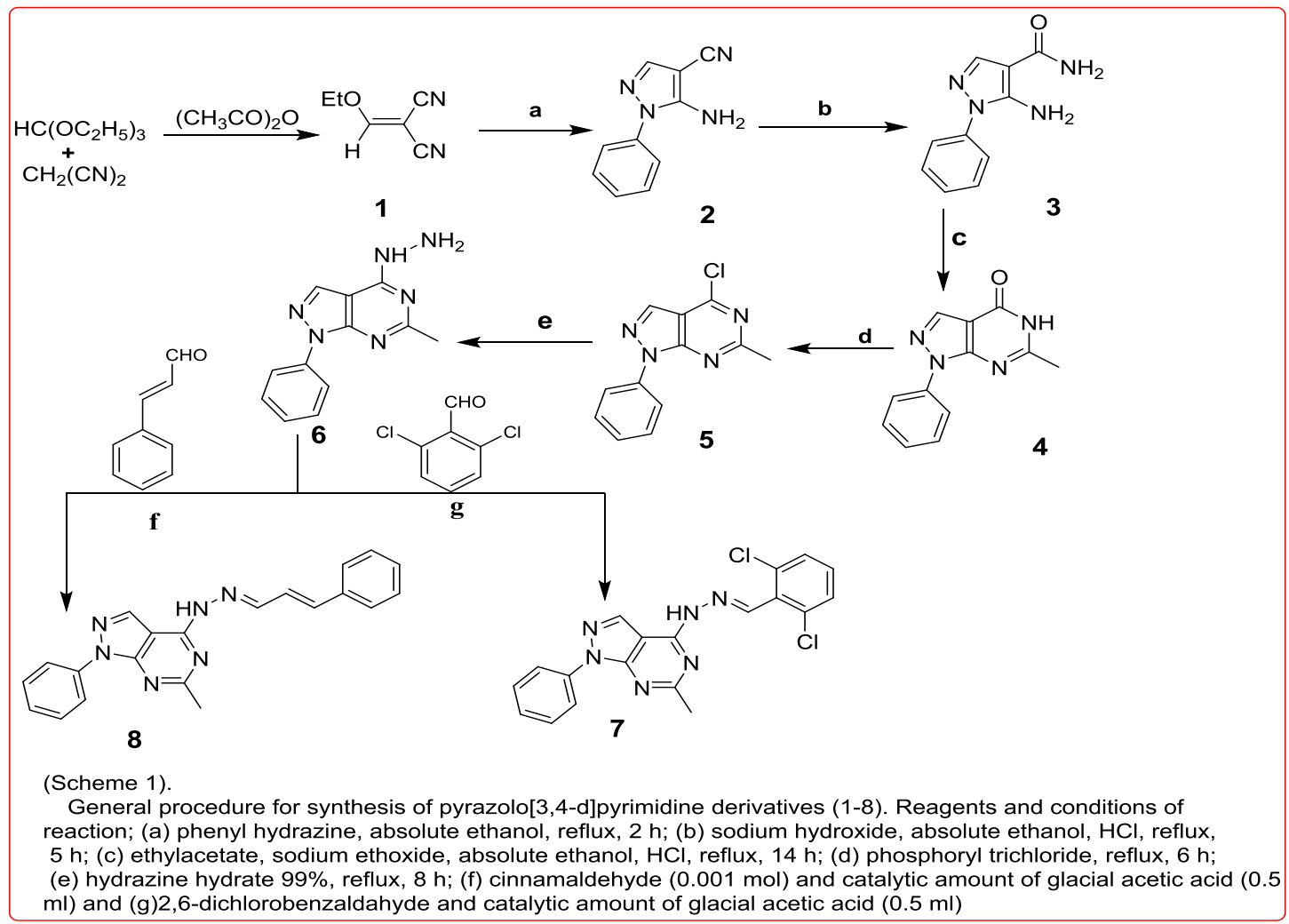

Thiole derivative (9) was synthesized from compound (4) through reflux with phosphorous pentasulfide in piperidine for 6 hours. The structure of compound (9) was confirmed by different analytical techniques as IR and $\mathrm{H}^{1} \mathrm{NMR}$. The $\mathrm{H}^{1} \mathrm{NMR}$ spectrum of compound (9) revealed singlet signal at $14.32 \mathrm{ppm}$ corresponding to (-SH)-proton.

For preparation of alkyl derivatives (10-13) were prepared by reaction of appropriate alkyl bromides, namely1-bromoisobutan, 1-bromononane, 1-bromo-3methylpentane, 1-bromo-2-ethylebutane with compound (9) in the presence of anhydrous potassium carbonate in DMF. This reaction proceeded smoothly and the desired compounds were obtained in good yields (68-75\%).(El-Gamal et al., 2015) 


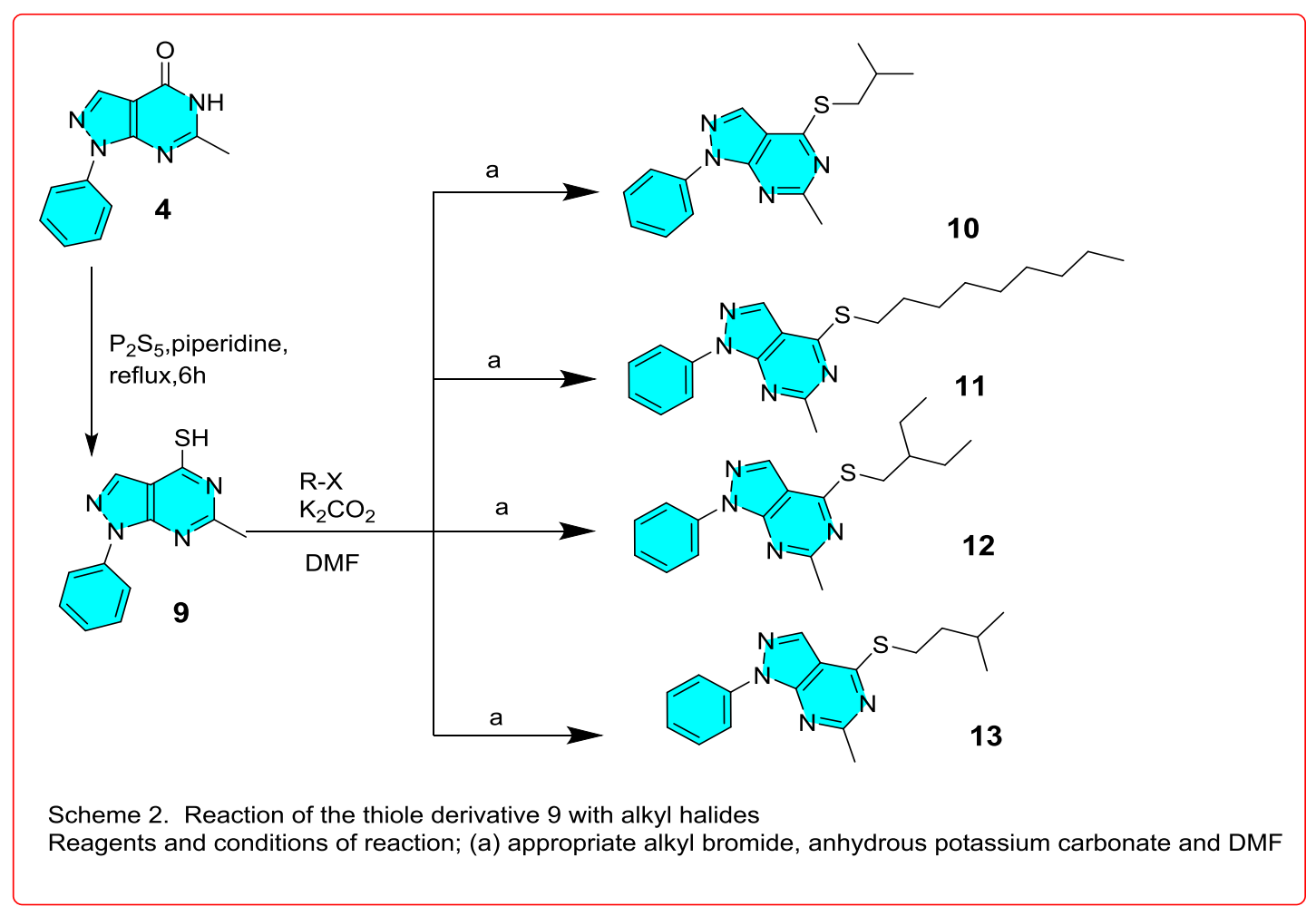

\section{II.2. Experimental.}

\section{II.2.1. General:}

Melting points were measured on a Gallen-kamp melting point apparatus and were uncorrected. The IR spectra were recorded on Nikolet IR 200 FT IR spectrophotometer using $\mathrm{KBr}$ discs $\left(\lambda\right.$ max in $\left.\mathrm{cm}^{-1}\right) . \mathrm{H}^{1} \mathrm{NMR}$ and $\mathrm{C}^{13} \mathrm{NMR}$ spectra were performed on Gemini 300BB spectrometer at $300 \mathrm{MHz}$ and Bruker spectrometer at $75 \mathrm{MHz}$, respectively. TMS was used as internal standard and DMSOd6 as solvent. The chemical shifts were reported in ppm $(\delta)$ and coupling constant $(J)$ values were given in Hertz (Hz). Signal multiplicities were represented by s (singlet), d (doublet), t (triplet), q (quadruplet), and $\mathrm{m}$ (multiplet). All of the new compounds were analyzed for $\mathrm{C}, \mathrm{H}$ and $\mathrm{N}$ and agreed with the proposed structures within $\pm 0.4 \%$ of the theoretical values by the automated CHN analyzer. Mass spectra were recorded on a unit of Shimadzu GCMSQP/MS-QP5050A spectrometer operating at $70 \mathrm{ev}$. The purity of the compounds was checked by thin layer chromatography (TLC) using Merck silica gel 60 F254 recoated sheets.

\section{General procedure for preparation of compounds (7) and (8).}

Amixture of hydrazine derivative (6) (0.30 g, 0.001 mol), 2,6dichlorobenzaldahyde, cinnamaldehyde $(0.001 \mathrm{~mol})$ and catalytic amount of glacial acetic acid $(0.5 \mathrm{ml})$ was heated under reflux in absolute ethanol $(20 \mathrm{ml})$ for specific time. The precipitate that formed on hot was filtered and crystallized from ethanol to yield the title compounds (7) and (8). 
4-(2-(2,6-Dichlorobenzylidene) hydrazinyl)-6-methyl-1-phenyl-1H-pyrazolo[3,4d]pyrimidine (7)

Red solid; reaction time: $6 \mathrm{~h}$; yield $83 \%(0.38 \mathrm{~g})$; m.p.: 255-257 ${ }^{\circ} \mathrm{C}$; IR (KBr, $v$, $\mathrm{cm}^{-1}$ ), 3383(NH), 3109 (Ar-H), 2910(aliph-CH). ${ }^{1} \mathrm{HNMR}\left(\mathrm{DMSO}-d_{6},\right): 8.55(\mathrm{~S}, 1 \mathrm{H},, \mathrm{NH}$, H4), 8.39 (s, 1H, phenyl-H), $8.24(\mathrm{~d}, 2 \mathrm{H}, J=7.3 \mathrm{~Hz}$, phenyl-H2, H6), $8.18(\mathrm{~s}, 1 \mathrm{H}-$ pyrazol), 7.60-7.65 (m, 3H, phenyl-H3, H4, H5), 7.39 (d, 2H, $J=7.3 \mathrm{~Hz}$, phenyl-H2, H6), 7.38 (m, 1H, phenyl-H5) , 2.33 (s, 3H, pyrimidine- $\left.\mathrm{CH}_{3}\right) .{ }^{13} \mathrm{C}$ NMR (DMSO, $d_{6}$ ): 165.9, 156.1, 154.8, 141.4, 139.1, 136.6, 134.1, 131.1, 131.6, 130.2, 129.9, 127.1, 126.8, 125.2, 99.4, 25.9. MS $(\mathrm{m} / \mathrm{z}): 398.28\left(\mathrm{M}^{+4}, 76.45 \%\right), 396.33\left(\mathrm{M}^{+2}, 100 \%\right)$; Anal. Calcd.for $\mathrm{C}_{19} \mathrm{H}_{14} \mathrm{Cl}_{2} \mathrm{~N}_{6}$ (397.26): C, 57.45; H, 3.55; N, 21.16. Found: C, 57.42; H, 3.51; $\mathrm{N}, 21.21$.

6-Methyl-1-phenyl-4-(2-((1E,2E)-3-phenylallidene) hydrazinyl)-1H-pyrazolo[3,4-d] pyrimidine (8)

Orange needle, reaction time: $5 \mathrm{~h}$; yield $95 \%(0.39 \mathrm{~g})$; m.p.: $241-243^{\circ} \mathrm{C}$; IR 7(KBr, $\left.v, \mathrm{~cm}^{-1}\right) .3317(\mathrm{NH}), 3112(\mathrm{Ar}-\mathrm{H}), 2886$ (aliph-CH). ${ }^{1} \mathrm{H}$ NMR (DMSO-d 6 ): 10.46 (s, 1H, NH, H4), 8.39 (d, 2H, $J=7.4 \mathrm{~Hz}$, phenyl-H2,H6), 8.18 (s, 1H-pyrazol), 7.94 (s, $1 \mathrm{H}, \mathrm{N}=\mathrm{CH}), 7.52-7.60(\mathrm{~m}, 3 \mathrm{H}$, ,phenyl-H3, H4, H5), 7.54 (d, 2H, $J=7.4 \mathrm{~Hz}$, phenyl-H2, H6), 7.33-7.45 (m, 3H, phenyl-H3, H4, H5), 7.22 (d, 1H, $J=7.5 \mathrm{~Hz}, \mathrm{CH}=\mathrm{C} \underline{\mathbf{H}}-\mathrm{ph}), 6.89$ $(\mathrm{d}, 1 \mathrm{H}, J=7.5 \mathrm{~Hz}, \mathrm{~N}=\mathrm{CH}-\mathrm{CH}=\mathrm{CH}), 2.35\left(\mathrm{~s}, 3 \mathrm{H}\right.$, pyrimidine- $\left.\mathrm{CH}_{3}\right) .{ }^{13} \mathrm{C}$ NMR (DMSO$d_{6}$, : 164.2 , 155.3, 154.5, 149.3, 139.2, 139.1, 137.4, 136.4, 129.8, 129.6, 127.6, 126.9, 125.1, 122.5, 99.5, 56.5, 25.5, 18.9. MS (m/z): $354.42\left(\mathrm{M}^{+}, 100 \%\right), 350.96(3.64 \%)$. Anal. Calcd.for $\mathrm{C}_{21} \mathrm{H}_{18} \mathrm{~N}_{6}$ (354.42): C, 71.17; H, 5.12; N, 23.71. Found: C, 71.14; H, $5.18 ; \mathrm{N}, 23.74$.

\section{6-Methyl-1-phenyl-1H-pyrazolo[3,4-d]pyrimidine-4-thiol (9)}

Phosphorus pentasulfide $(1.67 \mathrm{~g}, 3.75 \mathrm{mmol}$ ) was suspended in $40 \mathrm{ml}$ of pyridine and compound (4) $(0.5 \mathrm{~g}, 3.1 \mathrm{mmol})$ was added. The reaction mixture was refluxed for $5 \mathrm{~h}$. then $200 \mathrm{ml}$ of worm water was added to the contents of this flask. The precipitate was filtered off and recrystallized in petroleum ether to afford compound (9).

Yellow crystal; yield $91 \%(0.28 \mathrm{~g})$; m.p. $230-232^{\circ} \mathrm{C}$. IR $\left(\mathrm{KBr}, v, \mathrm{~cm}^{-1}\right): 3009(\mathrm{C}-$ $\mathrm{H}$ aromatic), 2933 (C-H aliphatic), $1498(\mathrm{C}=\mathrm{N}) .{ }^{1} \mathrm{HNMR}$ (DMSO-d $), 13.15$ (s, 1H, SH $\mathrm{D}_{2} \mathrm{O}$ exchangeable), $8.20(\mathrm{~d}, 2 \mathrm{H}, J=7.5 \mathrm{~Hz}$, phenyl-H2, H6), $8.16(\mathrm{~s}, 1 \mathrm{H}$, pyrazol), 7.52$7.60\left(\mathrm{~m}, 3 \mathrm{H}\right.$, phenyl-H3 , H4 , H5), 2.37 (s, 3H, pyrimidine- $\left.\mathrm{CH}_{3}\right) .{ }^{13} \mathrm{C}-\mathrm{NMR}$ (DMSO-d 6 ): $164.7,158.2,138.2,133.3,129.3,126.9,119.9,104.9,30.5,24.1 . \mathrm{MS}(\mathrm{m} / \mathrm{z}): 242.30$ $\left(\mathrm{M}^{+}, 75.74 \%\right), 241.62$ (100\%). Anal. Calcd. For $\mathrm{C}_{12} \mathrm{H}_{10} \mathrm{~N}_{4} \mathrm{~S}$ (242.30): C, 59.48; H, 4.16; N, 23.12. Found: C, 59.53; H, 4.19; N, 23.16.

\section{General procedure:}

Analogously to the previous scheme, compounds (10-13) were prepared by the reaction of alkyl bromide with compound (9) in the presence of anhydrous potassium carbonate $(0.001 \mathrm{~mol})$ in DMF. This reaction proceed smoothly and the desired compound DMF (20 ml) was heated under reflux for 10 hours then poured onto ice-cold 
water. The obtained precipitate was filtered, washed with water, dried and crystallized from ethanol to afford compounds (10-13), respectively.

\section{4-(Isobutylthio)-6-methyl-1-phenyl-1H-pyrazolo[3,4-d]pyrimidine (10)}

Yellwish crystals, $(2.2 \mathrm{~g} ; 76 \%)$; m.p. $80-83^{0} \mathrm{C}$; IR $(\mathrm{KBr}) \mathrm{cm}^{-1}: 3017$ (C-H aromatic), 2957 (C-H aliphatic), $1563(\mathrm{C}=\mathrm{N}) .{ }^{1} \mathrm{H}-\mathrm{NMR}$ (DMSO- $\left.d_{6}\right) \delta \mathrm{ppm:} 8.43(\mathrm{~d}, 2 \mathrm{H}$, $J=7.4 \mathrm{~Hz}$, phenyl-H2, H6), 8.20 (s, 1H-pyrazol), 7.61-7.57 (m, 3H, phenyl-H3, H4, H5), $4.12\left(\mathrm{~d}, 2 \mathrm{H}, J=7.4 \mathrm{~Hz}, \mathrm{~S}-\mathbf{C H}_{2}\right), 2.51(\mathrm{~s}, 3 \mathrm{H}$, pyrimidine-CH$), 1.05(\mathrm{~m}, 1 \mathrm{H}, \mathbf{C H}-$ $\left.\left.\left(\mathrm{CH}_{3}\right)_{2}\right), 0.91\left(\mathrm{~d}, 6 \mathrm{H}, J=6.8 \mathrm{~Hz}, \mathrm{CH}-\left(\left(\mathbf{C H}_{3}\right)_{2}\right) .{ }^{13} \mathrm{C} \text { NMR (DMSO-d }\right)_{6}\right): 166.2,163.6$, $155.5,139.0,133.3,129.7,127.0,121.3,101.9,54.5,40.5,39.8,28.5,26.1 . \mathrm{MS}(\mathrm{m} / \mathrm{z})$ : $298.41\left(51.53 \%, \mathrm{M}^{+}\right), 155.24(100 \%)$. Anal. Calc. for $\left(\mathrm{C}_{16} \mathrm{H}_{18} \mathrm{~N}_{4} \mathrm{~S}\right): \mathrm{C}, 64.40 ; \mathrm{H}, 6.08$; N, 18.78; Found: C, 64.45; H, 6.12; N, 18.90 .

\section{Methyl-4-(nonylthio)-1-phenyl-1H-pyrazolo[3,4-d]pyrimidine (11)}

Yellow crystals, $(2.20 \mathrm{~g} ; 70 \%)$; m.p. $85-95^{0} \mathrm{C}$; IR $(\mathrm{KBr}) \mathrm{cm}^{-1}: 3072(\mathrm{C}-\mathrm{H}$ aromatic), 2925 (C-H aliphatic), $1559(\mathrm{C}=\mathrm{N}) .{ }^{1} \mathrm{H}-\mathrm{NMR}$ (DMSO- $\left.d_{6}\right) \delta \mathrm{ppm}: 8.46(\mathrm{~d}, 2 \mathrm{H}$, $J=7.4 \mathrm{~Hz}$, phenyl-H2, H6), 8.17 (s, 1H -pyrazol), 7.37-7.60 (m, 3H-phenyl-H3, H4, H5), 3.37 (t, $2 \mathrm{H}, J=7.4 \mathrm{~Hz}, \mathrm{~S}-\mathrm{CH} 2), 2.49$ (s, 3H, pyrimidine- $\left.\mathrm{CH}_{3}\right), 125-1.77\left(\mathrm{~m}, 6 \mathrm{H},\left(\mathrm{CH}_{2}\right)_{3}\right)$, 1.23-1.45 (m, 6H- $\left.\left(\mathrm{CH}_{2}\right)_{3}\right), 0.86(\mathrm{t}, 3 \mathrm{H}, J=7.2 \mathrm{~Hz}) .{ }^{13} \mathrm{C}$ NMR (DMSO-d 6 ): 165.1, 164.8, 152.1, 152.1, 138.8, 133.7, 129.7, 127.1, 121.5, 111.5, 40.5, 40.3, 39.7, 31.7, 29.2, 29.0, 28.8, 26.63, 22.54, 14.38. $\mathrm{MS}(\mathrm{m} / \mathrm{z}): 368.54\left(21.52 \%, \mathrm{M}^{+}\right), 241.77(100 \%)$. (Anal. Calc. for $\left(\mathrm{C}_{21} \mathrm{H}_{28} \mathrm{~N}_{4} \mathrm{~S}\right)$ : C, 68.44; H, 7.66; N, 15.20; Found:C,68.41; H, 7.69; N, 15.24.

\section{$\underline{\text { 4-((2-Ethylbutyl)thio)-6-methyl-1-phenyl-1H-pyrazolo[3,4- } d] \text { pyrimidine (12) }}$}

Brownish crystals, $(2.20 \mathrm{~g} ; 72 \%)$; m.p. $85-90^{0} \mathrm{C}$; IR $(\mathrm{KBr}) \mathrm{cm}^{-1}: 3072(\mathrm{C}-\mathrm{H}$ aromatic), 2962 (C-H aliphatic), $1560(\mathrm{C}=\mathrm{N}) .{ }^{1} \mathrm{H}-\mathrm{NMR}$ (DMSO- $\left.d_{6}\right): \delta \mathrm{ppm} .8 .44(\mathrm{~d}, 2 \mathrm{H}$, $J=7.4 \mathrm{~Hz}$, phenyl-H2, H6), 8.16 (s, 1H -pyrazol), 7.36-7.59 (m, 3H-phenyl-H3, H4, H5), $3.37\left(\mathrm{~d}, 2 \mathrm{H}-J=7.3 \mathrm{~Hz}, \mathrm{~S}-\mathrm{CH}_{2}\right), 2.51\left(\mathrm{~s}, 3 \mathrm{H}\right.$, pyrimidine- $\left.\mathrm{CH}_{3}\right), 1.60\left(\mathrm{~m}, 5 \mathrm{H}, 2 \mathbf{C H}_{2}\right), 0.93$ $\left(\mathrm{t}, 6 \mathrm{H}, J=7.5 \mathrm{~Hz}, 2 \mathrm{CH}_{3}\right) .{ }^{13} \mathrm{C}$ NMR (DMSO-d $): 165.1,164.7,152.1,138.8,133.6$, 129.6, 127.1, 121.4, 111.5, 40.5, 40.3, 39.9, 39.7, 39.3, 31.7, 26.5, 25.3, 11.3; $\mathrm{MS}(\mathrm{m} / \mathrm{z})$ : $326.46\left(24.90 \%, \mathrm{M}^{+}\right), 226(100 \%)$. (Anal. Calc. for $\left(\mathrm{C}_{18} \mathrm{H}_{22} \mathrm{~N}_{4} \mathrm{~S}\right): \mathrm{C}, 66.22 ; \mathrm{H}, 6.79 ; \mathrm{N}$, 17.16; Found:C,66.25; H, 6.83; N, 17.19.

\section{$\underline{\text { 4-(Isopentylthio)-6-methyl-1-phenyl-1H-pyrazolo[3,4- } d \text { ]pyrimidine (13) }}$}

brownish crystals, $(2.23 \mathrm{~g} ; 75 \%)$; m.p. $80-87^{0} \mathrm{C}$; IR $(\mathrm{KBr}) \mathrm{cm}^{-1}: 3076(\mathrm{C}-\mathrm{H}$ aromatic), $2955\left(\mathrm{C}-\mathrm{H}\right.$ aliphatic), $1550(\mathrm{C}=\mathrm{N}) .{ }^{1} \mathrm{H}-\mathrm{NMR}\left(\mathrm{DMSO}-d_{6}\right) \delta \mathrm{ppm}: 8.47(\mathrm{~d}, 2 \mathrm{H}$, $J=7.4 \mathrm{~Hz}$, phenyl-H2, H6), 8.18 (s, 1H -pyrazol), 7.61-7.59 (m, 3H, phenyl-H3, H4, H5), $3.41\left(\mathrm{t}, 2 \mathrm{H}, J=7.4 \mathrm{~Hz}, \mathrm{~S}-\mathbf{C H}_{2}\right), 2.51\left(\mathrm{~s}, 3 \mathrm{H}\right.$, pyrimidine- $\left.\mathrm{CH}_{3}\right), 1.74\left(\mathrm{~m}, 2 \mathrm{H}, \mathbf{C} \mathbf{H}_{2^{-}}\right.$ $\mathrm{CH}-\left(\left(\mathrm{CH}_{3}\right)_{2}\right), 1.65\left(\mathrm{~m}, 1 \mathrm{H}, \mathbf{C H}-\left(\mathrm{CH}_{3}\right)_{2}, 0.95\left(\mathrm{~d}, 6 \mathrm{H}, \mathrm{J}=6.8 \mathrm{~Hz}, \mathrm{CH}-\left(\mathbf{C} \mathbf{H}_{3}\right)_{2}\right) .{ }^{13} \mathrm{C} \mathrm{NMR}\right.$ (DMSO- $\left.d_{6}\right)$; 164.7, 152.3, 138.8, 133.5, 129.6, 127.0, 121.2, 111.4, 40.3, 39.9, 38.2, 27.4, 26.5, 22.5. $\mathrm{MS}(\mathrm{m} / z): 312.44\left(21.58 \%, \mathbf{M}^{+}\right), 263.31(100 \%)$, Anal. Calc. for $\left(\mathrm{C}_{17} \mathrm{H}_{20} \mathrm{~N}_{4} \mathrm{~S}\right)$ : C, 65.35; H, 6.45; N, 17.93; Found:C,65.49; H, 6.50; N, 17.97. 


\section{4- Biological Evaluation:}

\subsection{In Vitro Anticancer Screening.}

According to the rational drug design, a series of novel pyrazolopyrimidine derivatives were designed and synthesized. Consequently, the new synthesized compounds were evaluated for their in vitro cytotoxic activity against four different cancer cell lines namely, hepatocellular carcinoma (HepG-2), human breast adenocarcinoma (MCF-7) and colorectal carcinoma (HCT-116) via standard MTT method (Mosmann, 1983; Denizot and Lang, 1986; Thabrew et al., 1997),

From the obtained anticancer results, it is evident that the screened compounds displayed different levels of cytotoxicity ranging from potent, moderate, weak, and inactive cytotoxicity against all tested cell lines. Therefore, data represented in (Table 1) revealed that, compound (9) was found to be significantly equipotent and efficient than doxorubicin with IC50 values of $4.50,4.17$ and $5.13 \mu \mathrm{M}$ against tested cell lines.Moreover, compound (9) was found to be the most potent derivatives against the three cell lines with IC50 values less than $30 \mu \mathrm{M}$ compared with an anticancer drug, doxorubicin as control. Besides, compound (10) possessed moderate anti-proliferative activities against the three cell lines with IC50 values ranging from $18 \mu \mathrm{M}$ to $40 \mu \mathrm{M}$. Furthermore, compound(13) showed weak anti-proliferative activities with IC50 values ranging from 75 to $96 \mu \mathrm{M}$. Finally, compounds $(\mathbf{7 , 8 , 1 1 )}$ and (12) appeared to be inactive against tested cell lines.

Table 1:In- vitro anti-proliferative activities towards HePG2, HCT-116 and MCF-7 cell lines.

-IC50 ( $\mu \mathrm{M}$ ): 1 - 10 (very strong). 11 - 20 (strong). 21 - 50 (moderate). 51 - 100 (weak)

and above 100 (non-cytotoxic)

••• DOX Doxoru:bicin

for IC50 values of the active compounds are summarized in Table 1.

$\underline{\text { Cytotoxic activity of some compounds against human tumor cells }}$

\begin{tabular}{|l|l|l|l|}
\hline & \multicolumn{3}{|l|}{ In vitro Cytotoxicity IC50 $(\mu \mathrm{M}) \bullet$} \\
\cline { 2 - 4 } & HePG-2 & MCF-7 & HCT-116 \\
\hline DOX & $\mathbf{4 . 5 0} \pm \mathbf{0 . 2}$ & $\mathbf{4 . 1 7} \pm \mathbf{0 . 2}$ & $\mathbf{5 . 2 3} \pm \mathbf{0 . 3}$ \\
\hline 7 & $\mathbf{1 0 0}>$ & $\mathbf{1 0 0}>$ & $\mathbf{1 0 0}>$ \\
\hline 8 & $\mathbf{1 0 0}>$ & $\mathbf{1 0 0}>$ & $\mathbf{7 6 . 8 4} \pm 4.1$ \\
\hline 9 & $\mathbf{2 7 . 8 3} \pm \mathbf{2 . 1}$ & $\mathbf{2 4 . 2 3} \pm \mathbf{1 . 6}$ & $\mathbf{1 2 . 0 9} \pm \mathbf{1 . 0}$ \\
\hline 10 & $\mathbf{5 4 . 7 9} \pm 3.6$ & $\mathbf{5 0 . 5 8} \pm \mathbf{3 . 2}$ & $\mathbf{3 5 . 4 8} \pm \mathbf{2 . 3}$ \\
\hline 11 & $\mathbf{1 0 0}>$ & $\mathbf{1 0 0}>$ & $\mathbf{1 0 0}>$ \\
\hline 12 & $\mathbf{1 0 0}>$ & $\mathbf{1 0 0}>$ & $\mathbf{1 0 0}>$ \\
\hline 13 & $\mathbf{7 5 . 2 6} \pm 4.2$ & $\mathbf{9 6 . 8 1} \pm 5.4$ & $\mathbf{1 0 0}>$ \\
\hline
\end{tabular}




\section{5-Conclusion:}

A series of novel pyrazolo[3,4- $d$ ]pyrimidine derivatives have been designed and synthesized in useful yields. All the new synthesized compounds were biologically screened in vitro for their cytotoxic activities against a panel of four cancer cell lines namely, HepG-2, MCF-7 and HCT-116.The results of cytotoxic evaluation indicated that compound (6) was found to be significantly more potent and efficient than doxorubicin with $\mathrm{IC}_{50}$ values of $4.50,4.17$ and $5.13 \mu \mathrm{M}$ against tested cellines Moreover, Pharmacophoric features indicated that pyrazolo[3,4-d]pyrimidine scaffold having a four atoms linker was more potent than those possessing other linkers which lead to significant decrease in cytotoxic activity.

\section{REFERENCES:}

Bailly, C. (2012). Contemporary challenges in the design of topoisomerase II inhibitors for cancer chemotherapy. Chemical reviews, 112(7), 3611-3640.

Cheng, C., \& Robins, R. K. (1956). Potential purine antagonists. VI. Synthesis of 1alkyl-and 1-aryl-4-substituted pyrazolo [3, 4-d] pyrimidines 1, 2. The Journal of Organic Chemistry, 21(11), 1240-1256.

Denny, W. A. (2004). Emerging DNA topoisomerase inhibitors as anticancer drugs. Expert opinion on emerging drugs, 9(1), 105-133.

Ding, R., He, Y., Xu, J., Liu, H., Wang, X., Feng, M., . . . Peng, C. (2012). Preparation and bioevaluation of $99 \mathrm{~m}$ Tc nitrido radiopharmaceuticals with pyrazolo [1, 5-a] pyrimidine as tumor imaging agents. Medicinal Chemistry Research, 21(4), 523-530.

Espinosa, E., Zamora, P., Feliu, J., \&Barón, M. G. (2003). Classification of anticancer drugs - a new system based on therapeutic targets. Cancer treatment reviews, 29(6), 515-523.

EL-ENANY, M. M. et al.( 2010) Synthesis and antitumor activity of novel 6-aryl and 6 alkylpyrazolo [3, 4-d] pyrimidin-4-one derivatives. European journal of medicinal chemistry, v. 45, n. 11, p. 5286-5291, . ISSN 0223-5234.

EL-GAMAL, K.; SHERBINY, F.; EL-MORSI, A. J. P. P. I. J. (2015) Design, synthesis and antimicrobial evaluation of some novel quinoline derivatives. v. 2 , n. 5 , p. $165-177$,

ELSEBAEI, M. M. et al. (2019) Lipophilic efficient phenylthiazoles with potent undecaprenyl pyrophosphatase inhibitory activity. v. 175, p. 49-62,. ISSN 0223-5234.

Gerber, D. E., (2008) Targeted therapies: a new generation of cancer treatments. Am Fam Physician, 77. 
He, H.-Y., Zhao, J.-N., Jia, R., Zhao, Y.-L., Yang, S.-Y., Yu, L.-T., \&Yang,L. (2011). Novel pyrazolo [3, 4-d] pyrimidine derivatives as potential antitumor agents: exploratory synthesis, preliminary structure-activity relationships, and in vitro biological evaluation. Molecules, 16(12), 10685-10694.

Huang, Z.-H., Zhuo, S.-T., Li, C.-Y., Xie, H.-T., Li, D., Tan, J.-H., . . Huang,S.-L. (2013). Design, synthesis and biological evaluation of novel mansonone $\mathrm{E}$ derivatives prepared via CuAAC click chemistry as topoisomerase II inhibitors. European journal of medicinal chemistry, 68, 58-71.

Huang, H., Chen, Q., Ku, X., Meng, L., Lin, L., Wang, X., . . . Li, M. (2010). A series of $\alpha$-heterocyclic carboxaldehyde thiosemicarbazones inhibit topoisomerase II $\alpha$ catalytic activity. Journal of medicinal chemistry, 53(8), 3048-3064.

Hurley, L. H. (2002). DNA and its associated processes as targets for cancer therapy. Nature Reviews Cancer, 2(3), 188.

Islam, M. S., Park, S., Song, C., Kadi, A. A., Kwon, Y., \& Rahman, A. M. (2017). Fluorescein hydrazones: a series of novel non-intercalative topoisomerase II $\alpha$ catalytic inhibitors induce G1 arrest and apoptosis in breast and colon cancer cells. European journal of medicinal chemistry, 125, 49-67.

Kaina, B. (2003). DNA damage-triggered apoptosis: critical role of DNA repair, double-strand breaks, cell proliferation and signaling. Biochemical pharmacology, 66(8), 1547-1554.

Laponogov, I., Veselkov, D. A., Crevel, I. M.-T., Pan, X.-S., Fisher, L. M., \& Sanderson, M. R. (2013). Structure of an 'open'clamp type II topoisomeraseDNA complex provides a mechanism for DNA capture and transport. Nucleic acids research, 41(21), 9911-9923.

Lee, J. H., Wendorff, T. J., \& Berger, J. M. (2017). Resveratrol: a novel type of topoisomerase II inhibitor. Journal of Biological Chemistry, 292(51), 2101121022.

Liu, L. F. (1989). DNA topoisomerase poisons as antitumor drugs. Annual review of biochemistry, 58(1), 351-375.

MANCY, A. et al. Balancing physicochemical properties of phenylthiazole compounds with antibacterial potency by modifying the lipophilic side chain. v. 6, n. 1, p. 80-90, 2019. ISSN 2373-8227.

Miyashita, A., Iijima, C., \&Higashino, T. (1990). Studies on pyrazolo [3, 4-d] pyrimidine derivatives. XVIII,Facile preparation of 1H-pyrazolo [3, 4-d] pyrimidin-4 [5H]-ones. Heterocycles, 31(7), 1309-1314. 
Miyashita, A., Suzuki, Y., Iwamoto, K.-i., \& HIGASHINO, T. (1998). Catalytic action of azolium salts. IX. Synthesis of 6-aroyl-9H-purines and their analogues by nucleophilic aroylation catalyzed by imidazolium or benzimidazolium salt. Chemical and pharmaceutical bulletin, 46(3),390-399.

Mlcochova, P., Caswell, S. J., Taylor, I. A., Towers, G. J., \& Gupta, R. K. (2018). DNA damage induced by topoisomerase inhibitors activates SAMHD1 and blocks HIV-1 infection of macrophages. The EMBO journal, 37(1), 50-62.

Nitiss, J. L. (2009). Targeting DNA topoisomerase II in cancer chemotherapy. Nature Reviews Cancer, 9(5), 338.

Pogorelčnik, B., Brvar, M., Žegura, B., Filipič, M., Solmajer, T., \&Perdih, A. (2015). Discovery of Mono-and Disubstituted 1H-Pyrazolo [3, 4] pyrimidines and $9 \mathrm{H}$-Purines as Catalytic Inhibitors of Human DNA Topoisomerase II $\alpha$. ChemMedChem, 10(2), 345-359.

Pommier, Y., Leo, E., Zhang, H., \& Marchand, C. (2010). DNA topoisomerases and their poisoning by anticancer and antibacterial drugs. Chemistry \& biology, 17(5), 421-433.

Schenone, S., Radi, M., Musumeci, F., Brullo, C., \&Botta, M. (2014). Biologically driven synthesis of pyrazolo $[3,4-d]$ pyrimidines as protein kinase inhibitors: an old scaffold as a new tool for medicinal chemistry and chemical biology studies. Chemical reviews, 114(14),7189-7238.

Shenkenberg, T. D., \& VON HOFF, D. D. (1986). Mitoxantrone: a new anticancer drug with significant clinical activity. Annals of internal medicine, 105(1), 6781.

Singla, P., Luxami, V., Singh, R., Tandon, V., \& Paul, K. (2017). Novel pyrazolo [3, 4-d] pyrimidine with 4-(1H-benzimidazol-2-yl)-phenylamine as broad spectrum anticancer agents: synthesis, cell based assay, topoisomerase inhibition, DNA intercalation and bovine serum albumin studies. European journal of medicinal chemistry, 126,24-35.

Sung,W.J.,Kim,D.H.,Sohn,S.K.,Kim,J.G.,Baek,J.H.,Jeon,S.B.,...Lee, K. B. (2005). Phase II trial of amsacrine plus intermediate-dose Ara-C (IDAC) with or without etoposide as salvage therapy for refractory or relapsed acute leukemia. Japanese journal of clinical oncology, 35(10), 612-616

Thun, M. J., DeLancey, J. O., Center, M. M., Jemal, A., \& Ward, E. M. (2010). The global burden of cancer: priorities for prevention. Carcinogenesis, 31(1), 100110.

Varrica, M. G., Zagni, C., Mineo, P. G., Floresta, G., Monciino, G., Pistarà, V., . . . Amata, E. (2018). DNA intercalators based on (1, 10-phenanthrolin-2-yl) isoxazolidin-5-yl core with better growth inhibition and selectivity than 
cisplatin upon head and neck squamous cells carcinoma. European journal of medicinal chemistry, 143, 583-590.

Wang, J. C. (2002). Cellular roles of DNA topoisomerases: a molecular perspective. Nature reviews Molecular cell biology, 3(6), 430.

Whittaker, S. R., Walton, M. I., Garrett, M. D., \& Workman, P. (2004). The Cyclindependent kinase inhibitor CYC202 (R-roscovitine) inhibits retinoblastoma protein phosphorylation, causes loss of Cyclin D1, and activates the mitogenactivated protein kinase pathway. Cancer research, 64(1), 262- 272.

Zhao, Y., Ge, C. W., Wu, Z. H., Wang, C. N., Fang, J. H., \& Zhu, L. (2011). Synthesis and evaluation of aroylthiourea derivatives of $4-\beta$-amino-4'-Odemethyl-4-desoxypodophyllotoxin as novel topoisomerase II inhibitors. European journal of medicinal chemistry, 46(3), 901-906

\section{تشييد وتقييم بيولوجي لبعض مشثقات البيرازولو(ب, ؟ ـد) بيريميدين الجديدة المتوقع لها نشاط مضاد للسرطان

$$
\text { 'وحبد علي زكي ، *'محمد السو اح ، 'محمد مصطفي السباعي، 'أثرف حسن بيومي }
$$

drmohammedalswah@azhar.edu.eg : البريد الالكتروني للباحث الرئيسي

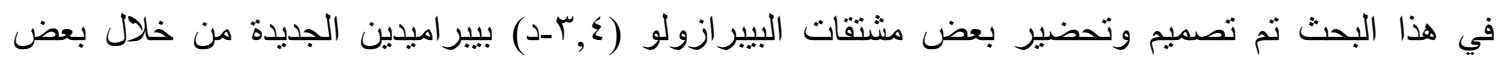

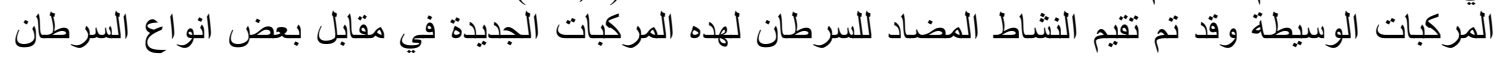

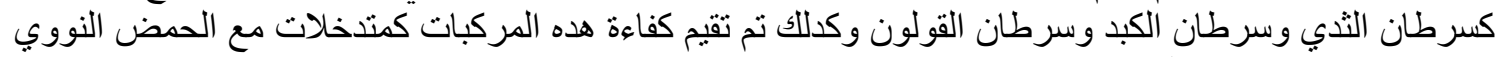

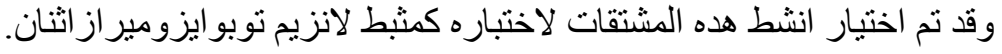

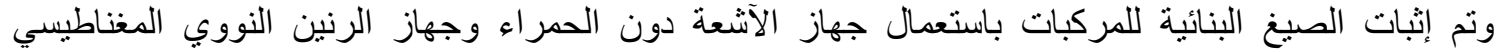

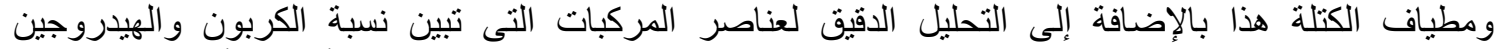

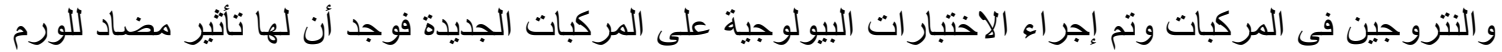

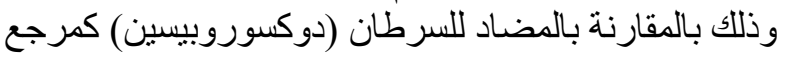

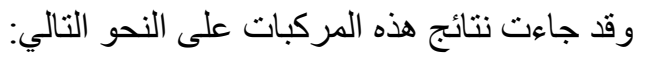

بالنسبة للنشاط المضاد للسرطان فقد كانت اقوى النتائج هي للمركب (9) حيث اظهر فاعلية كبيرة ضد جميع الخلايا وقد اظهرت نتائج تقييم السمية لهذه المركبات على النحو التالي، بالنسبة للنشاط المضاد للسرطان فقد كانت اقوى

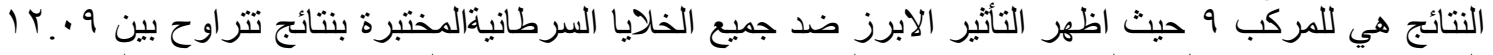

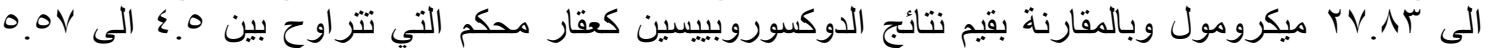

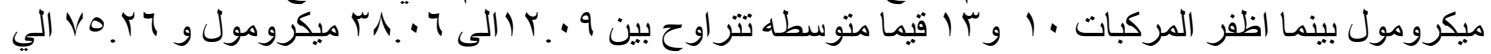

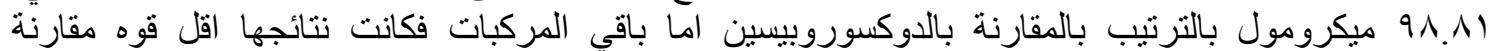
بالمركبات الاخرى.

الكلمات المفتاحية: مثبطات مستقبل عامل نمو البشرة، مشتقات البير ازولوبيريميدين ، مضاد للسرطان 